\title{
Articulação entre ensino e pesquisa no cuidado à saúde da criança: relato de experiência
}

\author{
Interaction between education and research in health care \\ child: experience report
}

Aline Cerqueira Santos Santana da Silva ${ }^{1}$ Érick Igor dos Santos ${ }^{2}$

\section{RESUMO}

Trata-se de um relato de experiência realizado com discentes do Curso de Graduação em Enfermagem de uma Universidade Federal, desenvolvido na unidade de internação da emergência pediátrica de um hospital público. Objetivo: Descrever a experiência da articulação entre ensino e pesquisa no cuidado à saúde da criança acerca das evidências cientificas de enfermagem no controle da infecção primária da corrente sanguínea, bem como prevenção e tratamento da infecção urinária. Método: Estudo descritivo, tipo relato de experiência. A busca foi realizada de agosto a dezembro de 2015, em bases de dados e na Biblioteca Virtual em Saúde. Resultado: Foi identificado que a infecção relacionada à assistência à saúde figura, na atualidade, como complicação prevalente entre as crianças hospitalizadas, destacando a infecção primária da corrente sanguínea, e a infecção do trato urinário. Conclusão: A articulação entre ensino e pesquisa possibilitou o levantamento de necessidades de saúde da criança no serviço de saúde, o compartilhamento de saberes por intermédio de debates, exposições dialogadas, workshops, seminários e o retorno aos profissionais do serviço sobre os problemas identificados e as soluções aplicáveis.

Palavras Chave: Ensino; Aprendizagem; Enfermagem pediátrica; Infecções urinárias; Infecções relacionadas a cateter.

\begin{abstract}
The aim of this paper is to provide an account of an experiment carried out by students from the nursing undergraduation course of a federal university, which was developed in a pediatric emergency of a public hospital. Objective: To describe the project experience concerning the scientific evidence on nursing in what comes to the control of primary blood stream infection and urinary infection, as well as its prevention and treatment. Method: Descriptive study in experiment report format. The research was performed from August to December of 2015 , at databases and at the Health Virtual Library. Result: The infection related to health assistance is currently portrayed as a prevalent complication among the hospitalized children, with highlights to the primary blood stream infection and urinary tract infection. Conclusion: The articulation between teaching and researching turned possible the identification of heath necessities children in the service heath, the knowledge share in debates, dialogued exhibitions, workshop and seminaries, the feedback for the service professionals about the identified problems and its solutions.
\end{abstract}

Key words: Teaching; Learning; Pediatric nursing; urinary tract infections; Infections related to catheter.

${ }^{1}$ Enfermeira Pediátrica pela Universidade Federal do Rio de Janeiro (UFRJ). Doutora e mestre em Enfermagem pela UFRJ. Professora da Universidade Federal Fluminense (UFF). Líder do grupo de pesquisa Estudos sobre vivências e integralidade dedicadas a enfermagem, criança, infância, adolescentes e recém-nascidos - EVIDENCIAR - UFF. Rio das Ostras, RJ, Brasil. E-mail: alinecer2014@gmail.com

${ }^{2}$ Enfermeiro Estomaterapeuta pela Universidade do Estado do Rio de Janeiro (UERJ). Doutor e mestre em Enfermagem pela UERJ. Professor da Universidade Federal Fluminense (UFF). Líder do Grupo de Pesquisa Laboratório sobre Enfermagem, Cuidado, Inovação e Organização da Assistência ao Adulto ou ao Idoso (LECIONAI) - UFF. Rio das Ostras, RJ, Brasil.E-mail: eigoruff@gmail.com 


\section{INTRODUÇÃO}

Este texto registra as experiências didático-pedagógicas na articulação entre ensino e pesquisa no cuidado à saúde da criança acerca das evidências cientificas de enfermagem no controle da infecção primária da corrente sanguínea (IPCS), bem como prevenção e tratamento da infecção do trato urinário (ITU) relacionada ao uso do cateter vesical de demora em crianças.

O projeto foi implementado ao longo da disciplina "Enfermagem no Cuidado à Saúde da Criança Hospitalizada" que engloba ensino teórico e prático, junto aos alunos de graduação do sétimo período acompanhados de docentes responsáveis pela disciplina, em um hospital público localizado na região dos lagos do estado do Rio de Janeiro.

Ao planejarem o projeto, os docentes vislumbraram estimular os estudantes à aquisição intra e extramuros da universidade e do hospital, do conhecimento sobre os riscos de saúde e morbidades que acometem a criança e, principalmente, fomentar o raciocínio crítico visando o entendimento e a importância dos cuidados prestados por enfermeiros enquanto protagonistas do cuidar das crianças e de suas famílias ali admitidas.

O conteúdo da disciplina foi organizado de maneira a instrumentalizar o aluno a prestar cuidados ao recémnascido e a crianças hospitalizadas, sob a égide dos anteparos éticos, doutrinários e legais da profissão e do Sistema Único de Saúde (SUS).

Este planejamento emergiu da observação feita em campo prático junto aos discentes, diante da assistência prestada pelo profissional enfermeiro à criança hospitalizada, onde, nesta unidade, a ITU e a IPCS apresentam-se como a principal causa de morbidade $e$ internação entre crianças de 0 a 5 anos de idade.

Estas crianças, quando admitidas, geralmente apresentam instabilidade clínica, necessitando de intervenções imediatas e prolongadas. Assim, a proposta terapêutica instituída em vigência destas morbidades dá-se, sobretudo, através da terapia intravenosa (TI), configurando-se, hoje, ação indispensável na medicina moderna.

Esta terapia pode ser instituída de diferentes maneiras e períodos, a depender do esquema proposto, do propósito da terapia instituída e do diagnóstico do paciente. Neste sentido, um dos maiores desafios ao sucesso da terapêutica implementada é a manutenção de uma via de acesso venoso segura e duradoura, contribuindo, assim, para o reestabelecimento das condições clinicas e físicas da criança ${ }^{1}$.

É fato que a infecção relacionada à assistência à saúde (IrAS) se apresenta na atualidade como a complicação mais prevalente entre os pacientes internados e, assim, apresenta-se como um grande e complexo desafio no âmbito mundial, mediante à grande variedade de intervenções diagnósticas e terapêuticas que são implementadas².
Nesta corrente de pensamento, o Centers for Disease $\mathrm{Control}^{3}$, relata que, com o grande advento tecnológico, a incidência da infecção hospitalar cresceu em velocidade espantosa, principalmente nas unidades de terapia intensiva neonatal e pediátrica, devido ao longo tempo de permanência de pacientes com esse perfil na unidade.

Desta forma, delimitou-se como objeto para este estudo evidências cientificas de enfermagem no controle da IPCS, bem como prevenção e tratamento da ITU na assistência prestada à criança hospitalizada. Determinou-se como questão norteadora: de quais bases técnicas e conceituais os enfermeiros se utilizam para prevenção e controle da IPCS, bem como prevenção e tratamento da ITU na criança hospitalizada? E, por objetivo, buscou-se descrever a experiência da articulação entre ensino e pesquisa no cuidado à saúde da criança acerca das evidências cientificas de enfermagem no controle IPCS, bem como prevenção e tratamento da ITU.

\section{MÉTODO}

Trata-se de um relato de experiência didáticopedagógica desenvolvido na unidade de internação da emergência pediátrica no hospital público supracitado. Esta unidade é composta por duas enfermarias e possui leitos destinados ao isolamento, e, nos casos de maior complexidade, o hospital conta com o serviço de central de regulação de vagas para referenciar essas crianças.

A turma era composta de 24 alunos divididos em dois grupos. Cada grupo assumiu o desenvolvimento técnicocientífico de uma temática. 0 projeto foi desenvolvido de agosto a dezembro de 2015.

Neste projeto, buscou-se aliar conhecimento científico à prática assistencial, fomentando, nos estudantes, a Prática Baseada em Evidências (PBE), visto que esta ação possibilita apropriação de novo conhecimento gerado a partir de pesquisas realizadas previamente ${ }^{4}$.

Neste mesmo contexto, a sistematização da assistência de enfermagem (SAE) foi aplicada com o objetivo de identificar o fenômeno observado e seu significado, possibilitando 0 atendimento das reais necessidades apresentadas 5 .

As cinco etapas inerentes a este projeto de ensino serão ilustradas na figura abaixo e descritas a seguir.

Inicialmente, o projeto de ensino foi apresentado aos estudantes em sala de aula, sendo esclarecidos seu objetivo e postulados teórico-filosóficos. Na primeira etapa, os estudantes foram conduzidos ao hospital e estimulados a traçar um diagnóstico situacional da realidade local, registrando os problemas reais ou em potencial que identificassem no cenário. Neste momento, duas morbidades assumiram destaque em virtude de sua gravidade e ameaça à vida dos pacientes pediátricos, quais sejam a IPCS e a ITU relacionada ao cateterismo vesical. 


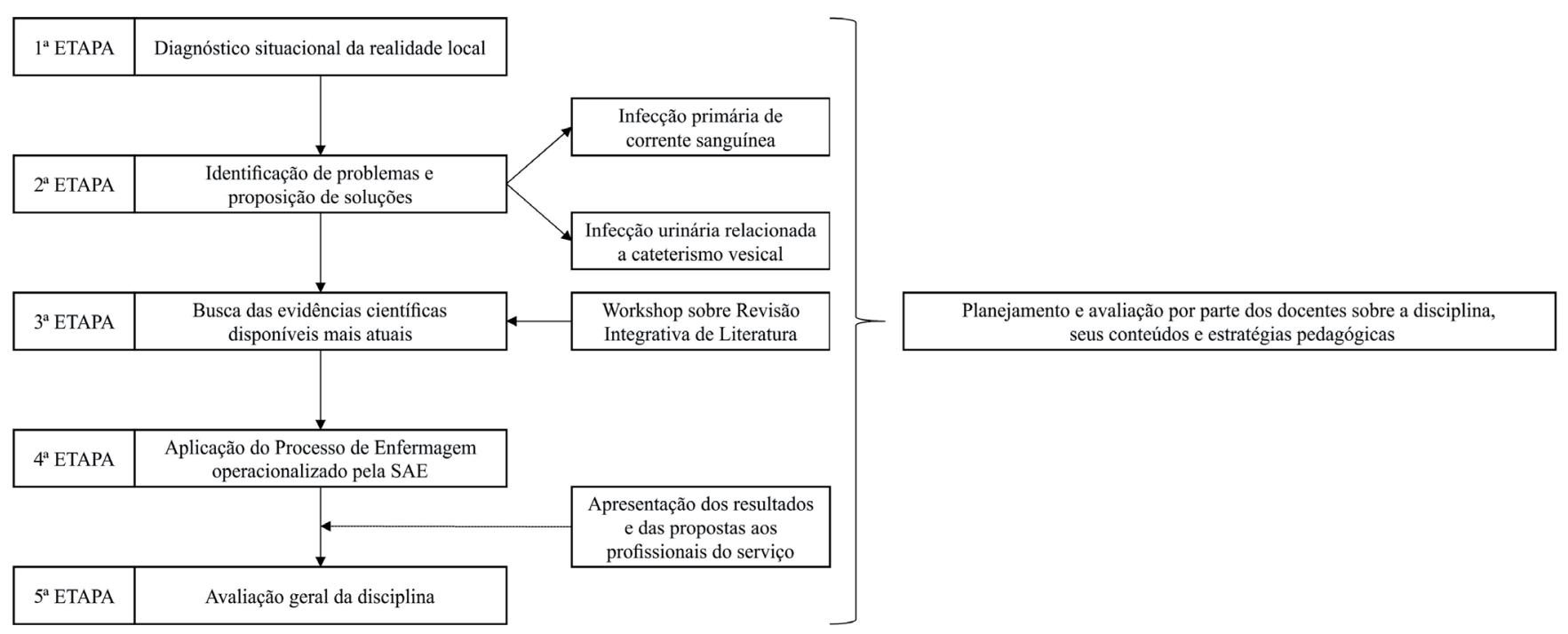

Figura 1- Fluxograma diagramático das etapas inerentes ao projeto de ensino- Rio das Ostras-RJ, Brasil.

$\mathrm{Na}$ segunda etapa, foram reunidas as propostas de soluções advindas do universo de saberes de estudantes e docentes. Esta busca por modos de atuar, foi propiciado ao aluno tornando-o sujeito ativo, construtor do conhecimento e compromissado com o cuidar e a docência. Tendo como propósito despertá-los para ação critica reflexiva, aliando o saber com o fazer, teoria-prática, visando o desenvolvimento de competências para a atuação no ensino, pesquisa e na assistência ${ }^{6}$.

A terceira etapa consistiu-se em uma apreciação das propostas elencadas ao crivo técnico-científico das evidências científicas disponíveis sobre os dois problemas identificados. Os estudantes participaram de um workshop, com duração de $10 \mathrm{~h}$ teóricas e práticas, ministrado pelos docentes sobre a Revisão Integrativa de Literatura (RIL), que se configura como método de pesquisa que permite a busca, a avaliação crítica e a síntese das evidências disponíveis do tema investigado, sendo o seu produto final o estado atual do conhecimento sobre o tema. Este produto possibilita, a identificação de lacunas que direcionam para o desenvolvimento de futuras pesquisas ${ }^{7}$.

Nesta vertente, os discentes foram instruídos a desenvolver o estudo através da revisão integrativa de literatura, percorrendo as seis etapas que concernem o método.

Nesse caminhar, o desenvolvimento do projeto permeou a realização de oficinas até a consecução do mesmo como proposta de aprendizagem compartilhada, visando a criatividade e a busca de soluções ${ }^{8}$.

Numa quarta etapa, os estudantes ficaram incumbidos de aplicar o Processo de Enfermagem sob referencial teórico de escolha, sendo operacionalizado pela Sistematização da Assistência de Enfermagem sob a taxonomia da North American Nursing Diagnosis Association (NANDA). Os problemas identificados nas dimensões biopsicoespirituais da criança e da sua família foram traduzidos em diagnósticos de enfermagem.
Em seguida, as Nursing Interventions Classifications (NIC) ou intervenções de enfermagem foram traçadas para minimizar os problemas identificados com base nas melhores evidências disponíveis. Para a redação das Nursing Outcomes Classifications (NOC), ou metas de enfermagem, os alunos foram orientados a planejar os resultados a serem alcançados pelos pacientes após as intervenções estabelecidas.

$\mathrm{Na}$ quinta etapa, após análise sobre as evidências, os alunos, organizaram-se ante a síntese do conhecimento, para apresentação das principais evidências de enfermagem encontradas acerca IPCS e ITU para os profissionais de saúde.

Nesta diretiva, os docentes organizaram um treinamento em serviço para apresentação dos resultados encontrados pelos alunos aos profissionais de saúde. Este treinamento objetivou a participação e o entendimento de cada profissional envolvido na prestação da assistência à criança hospitalizada, sobre os principais riscos e agravos apresentados durante a sua internação e quais medidas podem ser adotadas, como adoção de boas práticas para prevenção e controle de IrAS, a fim de assegurar o restabelecimento clínico e físico da criança livre de complicações.

\section{RESULTADO E DISCUSSÃO}

No que concerne à realização deste projeto de ensino, cujo objetivo foi descrever a experiência da articulação entre ensino e pesquisa com alunos de graduação no cuidado à saúde da criança acerca das evidências cientificas de enfermagem no controle da IPCS, bem como prevenção e tratamento da ITU, foi possível identificar dentre a análise das evidências cientificas atuais, sobre as temáticas em estudo, que a ITU representa de 35 a $45 \%$ de todas as infecções hospitalares ${ }^{9}$. E que a IPCS, na atualidade configura-se como um dos principais problemas 
de assistência à saúde, principalmente daqueles que necessitam, para sua recuperação clínica, da instalação e manutenção de um cateter venoso, seja ele central ou periférico ${ }^{10}$.

Sobre esta vertente, estima-se que cerca de $48 \%$ dos pacientes admitidos em UTI necessitam de dispositivos intravasculares, correspondendo a 15 milhões de cateter/ dia por ano, em que a densidade da incidência alcança 5,3 infecções relacionadas ao cateter por cada 1000 cateter/dia ${ }^{11}$.

Desta maneira, a mortalidade atribuível relacionada a essas infecções é de aproximadamente $18 \%$, correspondendo a 18 mil mortes/ano. Sem falar no fator de impacto negativo sobre o prolongamento no tempo de internação, em média por 7 dias e os custos atribuídos aos serviços de saúde ${ }^{11}$.

Neste ínterim, em 2005, tendo como foco o controle das IrAS o Comitê Consultivo de Práticas de Controle de Infecções Associadas à Assistência à Saúde (HICPAC), dos Estados Unidos, sob o consenso de suas entidades mais importantes relacionadas com o controle de IrAS, proclamaram a necessidade de os serviços de saúde começarem a trabalhar não só sobre os casos de infecção hospitalar, mas também, e principalmente, sobre os desfechos da assistência prestada ${ }^{12}$.

Nesta ótica, as IrAS têm grande potencial preventivo. Assim, ressaltamos as principais medidas de prevenção e controle da ITU evidenciadas: higienização adequada das mãos, manuseio correto do cateter, bem como do sistema de drenagem, padronização das técnicas, capacitação ou atualização periódica dos profissionais, monitorização de débito urinário, uso de sistema de drenagem fechado e orientação dos pacientes sobre a importância de sua higiene íntima ${ }^{13}$.

Em relação a IPCS, observa-se a adoção de boas práticas, como: implementação de um conjunto de medidas (Bundle) para inserção e manutenção do cateter venoso central (CVC), educação continuada, treinamento em equipe, envolvimento da equipe interdisciplinar e desenvolvimento de programas que avaliem assistência prestada, bem como acompanhamento pelo serviço de vigilância epidemiológica das ações realizadas na assistência, para controle e minimização de possíveis complicações ${ }^{12}$.

A imersão dos estudantes neste projeto, nos diferentes níveis de atenção à saúde, proporcionou amadurecimento acadêmico, somado a uma visão crítico-reflexiva, desenvolvendo a sensibilidade necessária para a promoção à saúde ${ }^{14}$

Nesta leitura e compreensão, os alunos estabeleceram uma aliança entre o saber e o fazer, certificando-se da necessidade da aproximação e conhecimento sobre as atuais evidências cientificas acerca do tema, identificando os fatores que intervêm diretamente na prestação de uma assistência de enfermagem segura e de qualidade implícita a qualquer clientela.
Desta maneira, a estratégia adotada para apresentação dos resultados encontrados nas atuais evidências cientificas, centrava-se na assertiva de que as infecções relacionadas à assistência à saúde (IrAS) ainda se configuram como uma complicação prevalente entre os pacientes internados. E que, dentre as avaliações processuais deste evento, algumas ações devem ser adotadas, tais como: ações de comunicação, acessibilidade, educação, investigações, prescrições, intervenções, entre outras ${ }^{12}$.

Compreende-se, assim, que o conhecimento dos fatores de risco e a conscientização e educação em serviço dos profissionais de saúde apresentem-se como medidas efetivas de prevenção e controle de infecção ${ }^{15}$.

\section{Avaliação do projeto de ensino}

Nesta perspectiva, acredita-se ser possível construir, através desta experiência, um espaço de reflexão e de compartilhamento de saberes, o que viabiliza a aprendizagem conjunta entre os participantes ${ }^{16}$.

Desta maneira, o ato de refletir e dialogar entre os pares no desenvolvimento de práticas torna possível formar profissionais de saúde com vistas a compreender a indissociabilidade entre ensino, pesquisa e extensão, resultando, assim, em novo perfil profissional ${ }^{15}$.

Assim, ocorreu, por parte dos alunos, o desvelar das necessidades inerentes ao serviço de saúde verificadas durante a prática desenvolvida. A participação no curso oferecido como uma das etapas iniciais do projeto favoreceu a oportunidade de partilhar suas dúvidas, apreender novos conhecimentos acerca do desenvolvimento de uma modalidade de pesquisa bem como o estabelecimento de uma relação dialógica entre docentes e discentes construção de novos conceitos elaboração de um espaço para construção e reconstrução das práxis, revisitando saberes e fazeres na assistência prestada por todos os profissionais envolvidos.

Ao final do período, docentes e discentes reuniramse para proceder à avaliação global da disciplina e realização do projeto. Por fim, destacam-se como pontos positivos no desenvolvimento deste projeto não só o amplo desenvolvimento dos alunos de graduação frente a nova modalidade de ensino aprendizagem, mas também o retorno ao serviço de saúde sobre as necessidades e as intervenções aplicáveis. Como produtos pode-se citar a realização do curso sobre revisão integrativa, confecção do material didático para apresentação oral e produção cientifica de artigos científicos (um para cada tema) seguida de apresentação em eventos científicos voltados para pediatria e neonatologia.

\section{CONCLUSÃO}

Conclui-se que a realização deste projeto em campo prático junto aos discentes permeou uma dialética que envolveu formas possíveis de cuidar, possibilitando 
identificar que a infecção relacionada à assistência à saúde figura, na atualidade, como uma complicação frequente entre os pacientes hospitalizados, destacando a IPCS, e a ITU como as mais prevalentes. A apropriação de novos conceitos, através dos estudos levantados e analisados constata que adoção de boas práticas na condução da assistência prestada tem grande potencial preventivo, contribuindo de forma positiva para a redução da morbimortalidade entre crianças hospitalizadas.

O desenvolvimento deste projeto aponta para novas direções na formação de profissionais, sendo possível aliar suas ações a uma visão critico-reflexiva tornando-os coparticipantes do pensar e do agir, do saber e do fazer. A metodologia adotada da PBE aliada ao levantamento de necessidades naquele cenário enfatizou a importância da atuação de cada discente com o papel social de transformar o seu entorno, de construir e reconstruir um modelo do sistema de saúde apoiado nos princípios da integralidade.

0 trabalho em grupo viabilizou a oportunidade de troca de saberes e práticas entre os estudantes, professores, além de profissionais da assistência direta em prol da construção de novos conhecimentos e oportunizando a refutação de antigos, o que permitiu à universidade uma intervenção pedagógica que não se limitou aos seus alunos, mas estendeu-se aos profissionais de saúde.

Apesar de o estudo ter alcançado o objetivo proposto, ainda possui limitações, como a baixa adesão dos profissionais de saúde no treinamento realizado para apresentação dos resultados da pesquisa. Por outro lado, esta pesquisa possui como potencialidade a estratégia pedagógica empregada, como campo fértil a ser semeado em outras disciplinas do curso, no intuito de articular saber e fazer rumo à prestação de assistência de enfermagem progressivamente mais crítica e reflexiva. Aponta-se também a necessidade de novos estudos na enfermagem sobre estas vertentes.

\section{REFERÊNCIAS}

1 - Dórea E, Castro TE, Costa P, Kimura AF, Santos FMG. Práticas de manejo do cateter central de inserção periférica em uma unidade neonatal. Rev Bras Enferm. 2011; 6(6):997-1002.

2 - Ferreira MVF, Andrade D, Ferreira AM. Controle de infecção relacionada a cateter venoso central impregnado com antissépticos: revisão integrativa. Rev Esc Enferm USP. 2011; 45(4):1002-1006.

3 - Centers For Disease Control. Bloodstream Infection Event (Central Line-Associated Bloodstream Infection and Noncentral line-associated Bloodstream Infection). [internet]. [publicado em 2014 jan; acesso em 2015 out. 3]. Disponível em: http://www.cdc.gov/nhsn/PDFs/pscManual/4PSC_ CLABScurrent.pdf.

4 - Medina EU, Pailaquilén RMB. A revisão sistemática e a sua relação com a prática baseada na evidência em saúde. Rev Latino-Am Enferm. 2010; 18(4):1-8.

5 - Silva MM, Moreira MC. Sistematização da assistência de enfermagem em cuidados paliativos na oncologia: visão dos enfermeiros. Acta Paul Enferm.2011; 2(2):172-178; 2011.

6 - Corrêa AK, Santos RA, Souza MCBM, Clapis MJ. Metodologia problematizadora e suas implicações para a atuação docente: relato de experiência. Educ Rev. 2011; 27(3):61-78.

7 - Santos Él. Cuidado e prevenção das skin tears por enfermeiros: revisão integrativa de literatura. Rev Gaúch Enferm. 2014; 35(2):142-149.

8 - Bastiani JAN, Padilha MICS. Experiência dos Agentes Comunitários de Saúde em Doenças Sexualmente Transmissíveis. Rev bras Enferm. 2007; 60(2):233-236.

9 - Souza ACS, Tipple AFV, Barbosa JM, Pereira MS, Barreto RASS. Cateterismo urinário: conhecimento e adesão ao controle de infecção pelos profissionais de enfermagem. Rev Eletr Enferm. 2007; 9(3):724-35.

10 - Mendonça SHF, Lacerda RA. Impacto dos conectores sem agulhas na infecção da corrente sanguínea: revisão sistemática. Acta Paul Enferm on line. 2010; 23(4):568-573.

11 - Calil K, Silvino ZR, Valente GSC. Bundle para manuseio do cateter venoso central: pesquisa exploratória e descritiva. Online Brazilian Journal Of Nursing. 2013; 12(0):713-15.

12 - Jardim JM, Lacerda RA, Soares NJD, Nunes BK. Avaliação das práticas de prevenção e controle de infecção da corrente sanguínea em um hospital governamental. Rev Esc Enferm USP. 2013; 47(1):38-45.

13 - Santos Él et al. Prevenção e controle da infecção urinária ao uso de cateter vesical de demora. Rev Enferm Atual In Derme. 2015; 11(73):23- 29.

14 - Sousa AR et al. Extensão universitária de enfermagem na atenção à saúde do homem: experiências em um cenário baiano. Rev Eletr Gestão Saúde. 2014; 5(esp):2709-2722.

15 - Moncaico ACS, Figueiredo RM. Conhecimentos e práticas no uso do cateter periférico intermitente pela equipe de enfermagem. Rev Eletr Enf on line. 2009; 11(3):620-7.

16 - Silva DM, Alves MR, Souza TO, Duarte ACS. Sexualidade na adolescência: relato de experiência. Rev enferm UFPE on line. 2013; 7(1):820-823. 